\title{
"Entretejiendo encajes" entre la universidad, los centros de arte y las escuelas
}

\section{"Interweaving laces" between the University, art centers and schools}

\author{
Gloria Jové Monclús \\ gjove@pip.udl.cat \\ Ester Betrián Villas \\ Facultad de Ciencias de la Educación. Departamento de Pedagogía y Psicología. \\ Universidad de Lleida \\ estherbetrian@pip.udl.cat
}

Recibido: 22 de septiembre de 2011

Aprobado: 7 de marzo de 2012

\section{Resumen}

Habitualmente la formación inicial de profesionales de la educación suele realizarse en los contextos universitarios, las escuelas son consideradas contextos para realizar las prácticas y los museos y centros de arte tienen poca presencia en dicha formación. Por ello desde la Facultad de Ciencias de la Educación de la UdL y en el contexto de la formación inicial de maestros y psicopedagogos empezamos a entretejer la red entre la Facultad, los centros educativos y los recursos culturales comunitarios para mejorar la formación de todas las personas implicadas. Concretamente, estamos desarrolando el espacio híbrido en el marco del proyecto Educ-arte dando especial énfasis a las relaciones creadas entre la Facultad de Ciencias de la Educación, el Centro de Arte la Panera y la escuela Príncep de Viana de Lleida. Para ello utilizamos el arte contemporáneo como mediador de aprendizajes y como instrumento de formación y construcción de conocimientos. Nuestra aportación está enmarcada en los planteamientos socioconstructivistas que sitúan la cultura como elemento básico para la humanización de las personas. Es por ello que en nuestro entretejer situamos los recursos comunitarios culturales en general y museos y centros de arte en particular como parte esencial del trabajo en red.

Palabras Clave: Espacio híbrido, arte contemporáneo, centro de arte, universidad, escuela.

Jové Moncús, G. y Betrián Villas, E. 2012: “Entretejiendo encajes” entre la universidad, los centros de arte y las escuelaso. Arte, Individuo y Sociedad, 24 (2), 301-314

\section{Abstract}

Usually the initial training of educational professionals often done at university contexts, the schools are considered contexts to do practices and museums and art centers are not taken into account in teachers training. Therefore, from the Faculty of Educational Sciences of UdL and in the context of initial training of teachers and advisory teachers began to weave the network between the Faculty, schools and community cultural resources to improve training of all involved. Specifically, we have been developing a hybrid space within the Educ-art project with special emphasis on the relationships created between the Faculty of Education, Panera art center and school Princep de Viana in Lleida. We use contemporary art as a mediator of learning and as training and to construct knowledge. Our contribution is framed in social-constructivist approaches that place culture as a basic element for the humanization of people. That is why in our interweaving we put the cultural community resources and museums and art centers in particular as an essential part of networking.

Key Words: Hybrid space, contemporary art, art center, university, school.

Jové Moncús, G. y Betrián Villas, E. 2012: “Interweaving laces” between the University, art centers and schools. Arte, Individuo y Sociedad, 24 (2), 301-314 
Sumario: 1. Sin patrones, 2. Bolillos, hilos, ganchitos y agujas, 3. Entretejiendo: la complejidad de los encajes, las agujas nos ayudan con la incertidumbre, 4. El encaje contemporáneo. Referencias.

El trabajo forma parte de una investigación más amplia que ha sido financiada por la Subdirección General de Proyectos de Investigación del Ministerio de Ciencia e Innovación, no 08669EDUC

\section{Sin patrones}

"Tradicionalmente los encajes se confeccionaban sobre un simple cojín, sin patrones, sólo con algunos bolillos y unos cuantos alfileres”.

Desde la Facultad de Ciencias de la Educación y en el contexto de la formación inicial de maestros y psicopedagogos empezamos a entretejer la red entre la Facultad, los recursos culturales comunitarios en general y los centros de arte y los museos en particular y los centros educativos y para mejorar la formación de todas las personas implicadas. Son habituales las situaciones donde la formación inicial de los profesionales de la educación se basa mayoritariamente en aspectos más teóricos y en el entorno universitario, el cual mantiene una hegemonía sobre la construcción y la difusión del conocimiento.

Las escuelas, por su parte, son simplificadas a un "lugar de práctica" en momentos puntuales (Barab y Duffy, 2000), obviando que tanto este entorno como otros entornos comunitarios pueden ofrecer muchas más posibilidades. Estamos de acuerdo con Zeichner (2010) en buscar nuevas epistemologías de la formación del profesorado donde el conocimiento académico no sea visto como la fuente autoritaria de conocimiento sobre la formación. Es necesario que los conocimientos existentes en las escuelas, en los museos y centros de arte y en los recursos comunitarios se trasladen a la formación del profesorado y coexistan en un plano de igualdad con el conocimiento académico que tradicionalmente se da en el campus. Huerta (2011) apuesta por los museos y por el arte contemporáneo como catalizadores de actividades educativas. Al conectar con las escuelas, se fortalecen, evolucionan y re-estructuran su coherencia con la sociedad. El enfoque contemporáneo creciente de re-pensar y re-diseñar la conexión entre el contenido de la universidad, los museos y centros de arte, las escuelas y las comunidades para las que el profesorado está siendo preparado para trabajar, ayudará a romper la desconexión que a menudo existe en el modelo tradicional de formación del profesorado. Para trabajar en esta dirección Zeichner (2010) propone los espacios híbridos. Pasar a la acción con proyectos concretos, con experiencias concretas es de vital importancia, evaluar, investigar y crear conocimiento en este ámbito es de vital relevancia.

Desde la Universidad de Lleida y en el marco del proyecto Educ-arte (Jové et. al., 2009) concretamos la formación inicial y permanente de maestros y psicopedagogos potenciando espacios y experiencias híbridas que permitan entretejer la red entre la Facultad, los recursos comunitarios y en especial los museos y los centros de arte (Jové, Betrián, Ayuso y Sansó 2010) y los centros educativos situando las formas de vida cultural como eje generador de aprendizajes y del desarrollo. Para formar profesionales de la educación que puedan dar respuesta a la heterogeneidad que hay 
en las aulas, los centros y en la sociedad del siglo XXI necesitamos que la formación inicial en la universidad esté interconectada con todo lo que pasa en la sociedad (Jové y Betrián, en prensa). Nuestra preocupación es cómo desde la formación de profesionales de la educación somos capaces de generar situaciones y crear espacios donde todos podamos aprender.

Actualmente y en el campo de la educación son muchas las voces que insisten en la necesidad de que los procesos educativos y de enseñanza se concreten en relación escuela-territorio, escuela-comunidad (Subirats, 1999; Gadner, 2001; Jové, Vicens, Cano, Serra y Rodríguez, 2006). Desde la formación de maestros y psicopedagogos, estamos concretando diferentes acciones y actividades para que los profesionales implicados se encuentren y realicen procesos en relación a la Facultad, los centros de arte y museos, la escuela y la comunidad. Como afirmaba Einstein, si queremos que pasen cosas diferentes tenemos que hacer cosas diferentes.

Esta mirada nos llevó en el 2001-2002 a iniciar el Proyecto Àlber (Jové, et al. 2006) en la escuela de Príncep de Viana, situada en la ciudad de Lleida. El Proyecto Àlber es un proyecto preventivo dirigido a toda la población basado en la construcción de las competencias básicas de acceso al currículo. La finalidad de este proyecto es que los alumnos tengan éxito y que aprendan a pensar y a convivir en la sociedad del siglo XXI. Pretendemos dar respuesta a la heterogeneidad de situaciones educativas que se dan hoy en día los centros educativos. La metodología parte del método como estrategia (Morin, Roger y Domingo, 2003), es decir, buscamos los recursos para trabajar con la incertidumbre y nos sirven para aprender, a la vez que generamos aprendizajes. Utilizamos diferentes estrategias organizativas y metodológicas siendo el eje vertebrador de los procesos de enseñanza-aprendizaje en el trabajo por proyectos, los agrupamientos multiedad e interniveles y el trabajo cooperativo y colaborativo, dando especial énfasis a la relación establecida entre entidades comunitarias a partir de los recursos que éstas nos ofrecen "local Cultural Project" (Moss, 2006). El proyecto se desarrolla en las aulas y el espacio híbrido. Se trata de un espacio de reflexión conjunta semanal entre todos los agentes implicados: todos los profesionales de la escuela, la profesora de la universidad, la educadora del centro educativo del centro de arte, el personal del centro de arte, los maestros y psicopedagogos en formación, los estudiantes de doctorado y otros agentes de los recursos comunitarios. Este espacio común para compartir y construir experiencias y conocimiento ha ido creciendo y reorganizándose como espacio híbrido, donde hemos ido concretando una estructura horizontal y un liderazgo compartido. A modo de ejemplo, ilustramos una interacción que se desarrolló en el espacio híbrido y que recogimos en el diario de notas durante la observación participante:

Los estudiantes de magisterio que hacen las prácticas en la escuela presentan una secuencia didáctica que han desarrollado los maestros tal y como ellos la han vivido:

Estudiante 1: Hemos recogido el proceso de desarrollo del "Proyecto maderitas" pero existen vacios porque no hemos visto cada día lo que pasaba ya que nosotros como practicantes veníamos un día a la semana. Partimos de la exposición Park Life de Martí Guixé en el Centro de Arte la Panera. Volvemos al aula y conversamos sobre lo que habiamos vivido, sentido... a partir de aqui devienen las actividades de enseñanza-aprendizaje. En el espacio híbrido lo compartimos, lo analizamos y 
aprendemos. Al escuchar las peticiones de los alumnos decidimos realizar una escultura conjunta con maderas. Cada niño tenía que traer una madera y personalizarla en función de sus características. Pero cuando los maestros realizan la propuesta, escuchan a los alumnos y están atentos al devenir del aula. Observan que los alumnos quieren personalizar su madera en función de sus propios criterios: como marco de fotos para una foto de su familia, como mástil para la bandera de su país,como la caña de pescar de su abuelo (véase Fig. 1).

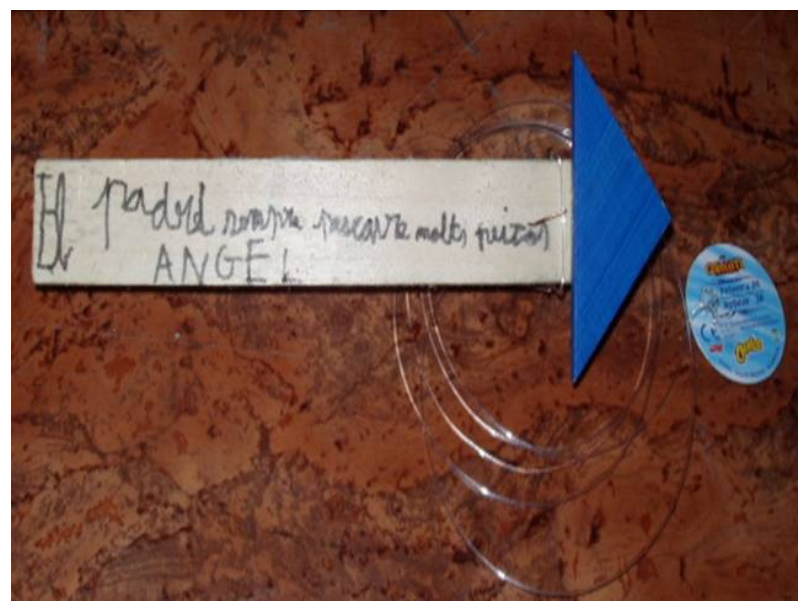

Figura 1. Producción del alumno en la que utiliza la madera como base para construir la caña de pescar.

Una vez personalizadas las maderas, las sacamos al patio y creamos una escultura entre todos (Fig. 2).

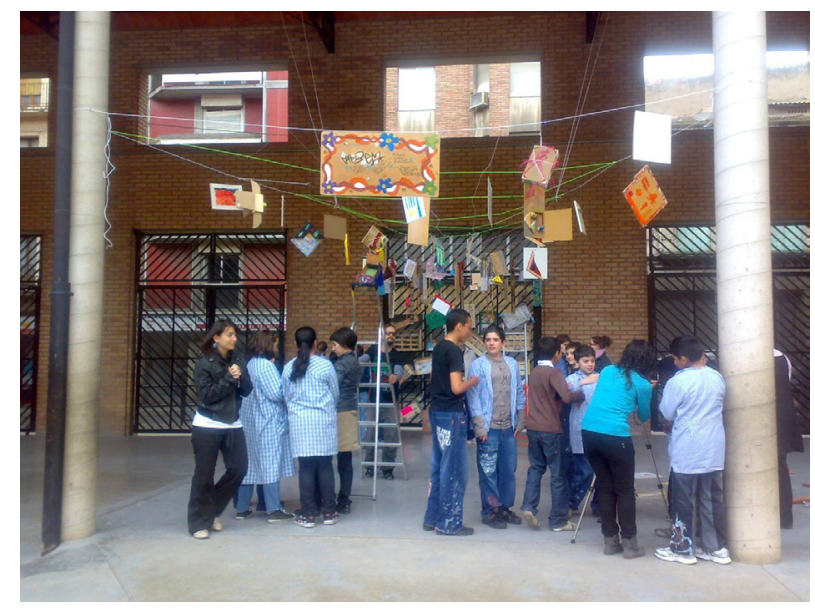

Figura 2. Escultura creada por todos los implicados en la escuela Príncep de Viana. 
Volvemos a la Panera a visitar la exposición de Javier Peñafiel "Voz entre líneas" y hay una escultura de Gustavo Marrone. Alan dice "son como nuestras obras". (Fig. 3).

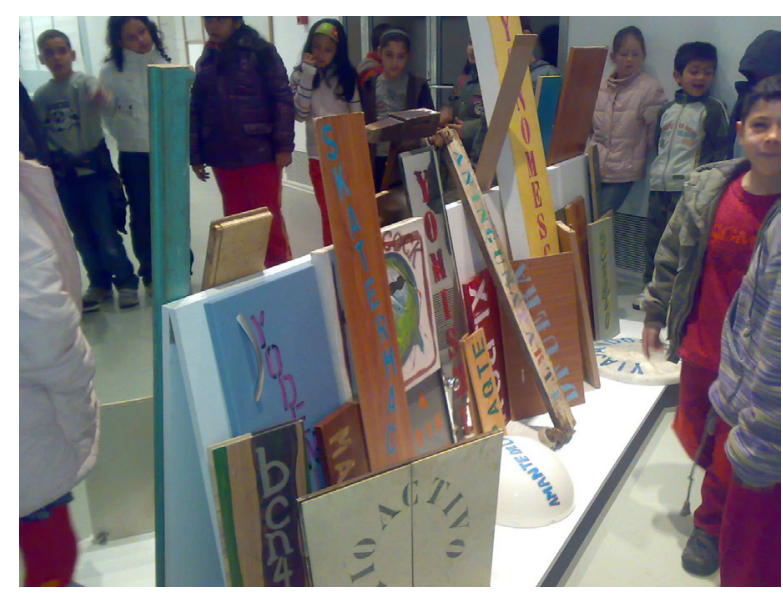

Figura 3. Visita a la exposición de Gustavo Marrone y relación de los niños con sus producciones.

Después de la exposición de la secuencia didáctica en el espacio híbrido, las estudiantes sugieren diferentes palabras e invitan a los asistentes del espacio híbrido a que elijan una palabra y verbalicen una frase sobre el proceso de enseñanza y aprendizaje. Algunos de los comentarios fueron:

Profesora de la Universidad: "Identidad. Partimos de la identidad, buscándola hemos encontrado la diferencia".

Maestro 9: "Trabajo en red. La coordinación con la Panera y otra gente ayuda. Los niños se han sentido artistas".

Estudiante 9: "Sentimiento de pertenencia de estudiantes, alumnos..."

Es un momento formativo donde son los estudiantes futuros maestros quienes nos muestran aquello que han vivido en los diferentes contextos y lo hacen público para comprender y reflexionar sobre el proceso. En los trabajos escritos los estudiantes lo definen como un espacio donde se reflexiona sobre lo que hacemos en las situaciones de enseñanza-aprendizaje para comprender lo que pasa y cómo mejorarlo. Esta estructura de prácticas posibilita el espacio formativo para todos los agentes implicados y donde podemos aprender y mejorar lo que estamos haciendo para que se mueva en el plano de la consistencia y la coherencia entre lo que decimos y los que hacemos y lo que mostramos.

En el curso 2001-2002, y en el marco de los estudios de magisterio, los profesores implicados en las materias troncales de la titulación de maestro-Educación Especial creamos una bolsa de créditos prácticos y los estudiantes de $2^{\circ}$ tenían que ir a un contexto escolar un día a la semana, con el fin de conocer y analizar el funcionamiento de las aulas de educación infantil y primaria. Uno de estos contextos educativos es la escuela Príncep de Viana. El grupo docente considera los contextos prácticos y 
el prácticum como el eje vertebrador de los procesos de innovación y cambio en la docencia. Pretendemos que los estudiantes en formación realicen procesos de interrelación, que construyan puentes, conexiones y que lo integren en su ser. Poco a poco vamos aprendiendo a explorar nuevas formas para ir más allá de la división entre la teoría y la práctica (Lenz-Taguchi, 2010) y que los estudiantes entretejan todas aquellas experiencias, vivencias y aprendizajes en el proceso formativo.

En este mismo curso 2001-2002 y en el marco de la asignatura "Dificultades de aprendizaje e intervención psicopedagógica" de los estudios de psicopedagogía, se ofrece a los estudiantes la posibilidad de realizar prácticas en diferentes entidades comunitarias de la ciudad de Lleida, en función de los intereses y perfiles de los estudiantes y los proyectos que han surgido del trabajo entre la Facultad y las entidades comunitarias. Las entidades con las que se han creado nexos de unión más intensos son los museos en general, y los centros de arte en particular.

El espacio híbrido que presentamos tiene un objetivo común: mejorar la calidad de la educación para contribuir al proceso humanizador y a la calidad de vida de las personas a través de la cultura y especialmente a través del arte, adquiriendo un papel protagonista el arte contemporáneo.

¿Por qué a través del arte? Porque según Eisner (2004) el arte permite desarrollar y explorar partes de la mente que nos ayudan a la transformación de la conciencia. El arte como estrategia, el arte como experiencia (Dewey, 2008). El arte es mediador de aprendizajes y un poderoso instrumento de formación y construcción de conocimiento, ya que nos habla del hoy y del ahora desde diferentes perspectivas. O'Sullivan (2006) considera el arte como potenciador de posibilidades y de mundos posibles y como una estrategia para poder desarrollar pensamientos rizomáticos (Deleuze y Guattari, 1987). Esto autores se refieren al rizoma como aquella estructura que carece de centro y donde los elementos pueden estar interconectados e influenciados entre sí. Responde a la lógica del cambio, la heterogeneidad y la multiplicidad. Es por ello que el arte plantea múltiples interrogantes, propone distintos horizontes, contribuye a la formación de individuos receptivos, críticos, dialogantes, imaginativos y reflexivos. Asimismo, se aprovecha la riqueza de los recursos de la comunidad para "salir" del currículo y de las asignaturas como disciplinas que muestran una realidad parcelada (Morin et al., 2003).

Empezamos con el proceso de apertura del aula y facilitamos el intercambio entre diversos contextos, como la Facultad de Ciencias de la Educación, los centros de arte y las escuelas de educación infantil y primaria. Además, el arte contemporáneo es una valiosa herramienta que permite emerger la heterogeneidad y que se caracteriza por una amplia transversalidad conceptual y transdisciplinaria. En resonancia, el arte es un medio que nos permite construir competencias creativas por un lado, para afrontar y gestionar nuevas situaciones, nuevos retos e incertidumbres, y por otro, para ir forjando nuestro camino con el continuo desarrollo de estrategias en un bucle recursivo que tiende al infinito. Por tanto creemos que el arte contemporáneo es una estrategia para la humanización y la calidad de vida. Aprendemos a través del arte. 


\section{Bolillos, hilos, ganchitos y agujas}

"Para entretejer se necesita bolillos, hilos, ganchitos y agujas".

¿Qué instituciones, recursos comunitarios, interacciones y estrategias se entretejen en la red del Proyecto Educ-Arte? En 2003, el Centro de Arte La Panera de Lleida, nace como plataforma de producción, difusión, formación y exhibición de las artes visuales en nuestro país. Pretende ser un instrumento que establezca puentes entre la creación visual y la creación producida en otros ámbitos culturales. Asimismo, con las exposiciones y las actividades que genera, trata de ser un centro de reflexión y adopta un compromiso con la sociedad que vincula las artes visuales con los diferentes sucesos de nuestra sociedad.

Desde su inicio, el Servicio Educativo de la Panera propone formación a todos los públicos, dando la posibilidad de conocer y disfrutar de la creación contemporánea a todos aquellos interesados. El objetivo de la propuesta educativa es que ante la heterogeneidad (culturas, edades, géneros, capacidades, facilidades, dificultades...) que nos rodea seamos capaces de establecer un nexo de unión que nos identifique a todos en una sola comunidad más rica en experiencias, convivencias y nuevos aprendizajes.

Empezamos a entretejer la red en el 2001-2002, y es a partir del curso 2004-05 que se ofrecen créditos prácticos a los estudiantes de Psicopedagogía en el Centro de Arte La Panera, cuyo objetivo es definir el papel del psicopedagogo en el servicio educativo de un centro de arte contemporáneo. Hasta este momento íbamos con algunos bolillos y unos cuantos alfileres. En el curso 2005-2006, a raíz de una visita de supervisión de prácticas por parte de la profesora de la UdL y una conversación con las educadoras del centro de arte, emergen con fuerza los bolillos, los hilos, los ganchitos y las agujas. Se concreta el trabajo en red creando interfaces entre el taller de robótica de Daniel Canogar y el proyecto de la escuela basado en la película "Robots".

\section{Entretejiendo: la complejidad de los encajes, las agujas nos ayudan con la incertidumbre}

"Los bastones hechos de madera de boj se utilizan para almacenar los hilos mientras se trabaja, y permiten trenzar el hilo creando los diferentes puntos. Las agujas son necesarias para que encaje se aguante”.

¿Cómo hemos desarrollado del proyecto? ¿Qué espacios híbridos se han concretado? ¿Cómo estamos entretejiendo la red? A partir del curso 2006-2007 entretejemos la red en el marco del Proyecto Educ-arte y a partir de la exposición "Glaskultur, ¿qué pasó con la transparencia?”, comisariada por Martí Peran. Aquel curso 2006-2007 el gobierno de Cataluña incorporó una hora más en la jornada educativa de primaria, la sexta hora y desde las áreas de sensibilidad artística acordaron contextualizar el currículum con la exposición C3, Colección de arte contemporáneo del Ayuntamiento de Lleida en la que figuraban artistas como Daniel Canogar, Alberto Peral, Marina Núñez, MP \& MP Rosado... entre otros. La última exposición que la escuela visitó durante aquel curso académico fue "+ Insectos" del artista Francisco Ruiz de Infante. El contenido se vinculó a las áreas de conocimiento del medio y de lengua. Tercero y 
cuarto de primaria se aproximaron a la exposición desde el área de lengua extranjera, inglés. Poco a poco el entusiasmo por el proyecto llevó a que vinculáramos las tres exposiciones que a lo largo del curso 2006-2007 se desarrollaran en la Panera con el currículo de la escuela. Fue entonces cuando los profesionales del centro de arte empezaron a asistir a alguna de las sesiones del espacio híbrido, y así comenzó a gestarse el rol de los educadores del centro de arte La Panera en el espacio híbrido de la escuela. Íbamos entretejiendo la red desde el contexto escolar.

Al principio todos tuvimos una gran sensación de satisfacción, sentimiento que a lo largo del curso se transformó en sobrecarga de trabajo (presión, tensión...). Demasiadas exposiciones, demasiado programado. No habíamos respetado uno de los principios básicos de nuestra metodología: estar muy atentos al devenir de la vida de las escuelas y de la comunidad. Lo habíamos programado desde inicio de curso y eso entraba en contradicción con nuestra manera de trabajar, donde el momento interactivo y postactivo, de revisión y análisis de la situación de enseñanza-aprendizaje adquiere mucha más importancia que el momento preactivo.

A partir de procesos de reflexión-acción nos planteamos revisar la metodología de trabajo. Durante el curso 2007-2008 planteamos un sistema de trabajo y organización más acorde con nuestra metodología. Se partió de una sola exposición, Mediterránea (as), y a medida que se iban desarrollando los proyectos se incorporaban otras exposiciones u obras concretas de artistas. Y ello permitió consolidar el papel del Centro de Arte en el espacio híbrido. En el curso 2007-2008 se constata que en estas sesiones no hay una demanda por parte de los docentes a los profesionales del centro de arte, sino que se genera un trabajo transversal y en red, que permite reconstruir el proyecto por parte de todos los profesionales que están en el espacio híbrido, ya sean maestros, profesionales del centro de arte, profesores de la UdL y / o estudiantes.

Durante estos años los estudiantes futuros maestros accedían y trabajaban mediante el arte en las escuelas implicadas en el proyecto. Todo lo observábamos, lo investigábamos en las escuelas y con las escuelas, en las instituciones y con los estudiantes. Pero en nuestras clases de la universidad continuábamos generando aprendizaje con casos, textos y contextos que tradicionalmente se han vinculado con el ámbito disciplinar de la psicopedagogía. El proyecto era común, estaba allí, y sonaba constantemente en nuestro entorno, pero la música no sonaba igual escuela, el centro de arte y los recursos comunitarios que en nuestras aulas universitarias. En los otros contextos había armonía, en nuestras aulas universitarias faltaba. Hasta el curso 2007-2008 los estudiantes vivían el proyecto como estudiantes en prácticas pero no por el trabajo que promovíamos desde la universidad.

El profesorado universitario que formamos maestros investigamos y decimos mucho sobre los procesos que realizan los demás, en sus clases, con sus alumnos y cómo deberían concretarse prácticas de calidad.... pero es menos frecuente que nuestro punto de investigación sea nuestra propia práctica, nuestra propia docencia, el análisis de las situaciones y de las comunidades prácticas de aprendizaje que somos capaces de generar para que sean espacios en los que podamos construir conocimiento y desarrollar procesos formativos de calidad. Revisamos nuestra docencia y la reorganizamos para generar aprendizajes, no sólo a través de textos y contextos propios de nuestro 
campo disciplinar, sino a través de recursos comunitarios y en especial del arte, en el marco del proyecto Educ-arte.

Así, el curso 2008-2009 coincidió con la conmemoración del 800 aniversario del nacimiento del Rey Jaume I. Se propuso a las escuelas aproximarse curricularmente al personaje a partir de las propuestas culturales que se realizaban en la ciudad y en el territorio. Hubo 23 escuelas que participaron, visitaron las exposiciones, concretaron aprendizaje en las aulas y algunas de ellas crearon una obra que fue expuesta en la Canonja, en la Seu Vella, donde casi 800 años atrás y a la edad de seis años fue proclamado rey Jaume I. La escuela Príncep de Viana decidió iniciar el proyecto con la salida a una exposición que se realizó al Instituto de Estudios Ilerdenses "Escenas imaginarias de la vida de Jaume I", de Antoni Borrell. Por tanto, aunque el contexto de aprendizaje inicial no era una exposición del Centro de arte La Panera, los profesionales del centro de arte realizaron el espacio híbrido conjunto y fuimos creando puentes entre esta exposición y otras obras del centro de arte La Panera y otros museos. Siempre en función del devenir del proyecto.

En este mismo curso 2008-2009 la asignatura "Aspectos didácticos y Organizativos" comenzó con la exposición "Interfaces", una selección de la colección de arte contemporáneo de la Fundación Sorigué (Lleida) y del Ayuntamiento de Lleida, que se llevó a cabo en el Centro de Arte La Panera. Los futuros maestros visitaron la exposición y se organizaron en cinco grupos para crear diálogos entre las obras sobre lo que más les sorprendía y lo que les sugería. Los autores que consideraron para trabajar cada grupo fueron Alicia Framis, Juan Pablo Ballester, Gary Hill, Antoni Abad y Juan Muñoz. Con la reflexión emergieron conceptos relacionados con los contenidos de la asignatura: identidad, etiquetaje, heterogeneidad, alteridad y construcción de la identidad personal y profesional.

Y la exposición se convirtió en una interfaz, un cruce atravesado por los puentes conceptuales que se fueron entretejiendo entre las obras y que hicieron posible, como decía Heidegger, que las orillas, es decir, las obras-emergieran como tales sólo cuando los puentes cruzaban las corrientes (Jové, et al. 2010). Los conceptos que emergieron se fueron desarrollando y plasmando en un mapa conceptual que se iba modificando a medida que avanzaban en su proceso. A continuación cada grupo de futuros maestros profundizó en un tema y expusieron su trabajo concretando una actividad en relación a un recurso comunitario con el fin de generar aprendizajes de servicio en el marco del proyecto de Jaume I.

Durante el 2009-2010 el contexto de aprendizaje inicial fue la exposición "Puertas Abiertas". Se trata de una colectiva de las obras que los niños de diferentes escuelas muestran a la comunidad sobre aquello que han trabajado durante el curso 2008-2009 a partir de las exposiciones del Centro de Arte la Panera. Allí había obras de la escuela Príncep de Viana del proyecto de Jaume I. Es una experiencia que permitió acceder al arte mediante las producciones artísticas de los alumnos de algunas escuelas donde los estudiantes realizan el prácticum de magisterio. Esta situación nos permite saborear la gestión de la incertidumbre como posibilidad que facilita el devenir del proceso de enseñanza-aprendizaje. Sería mucho más fácil realizar este proceso en un museo de exposición permanente. Cada curso podríamos realizar la misma secuencia, la misma actividad y devenir incertidumbre para nuestros alumnos, pero no para 
nosotros. Así que decidimos iniciar la asignatura en el mismo contexto pero con una exposición muy distinta a la del año anterior.

Observamos diferentes producciones que habían partido de la misma obra de arte. ¿Por qué se habían concretado de manera diferente? Los estudiantes formularon hipótesis e investigaron cómo se habían concretado las propuestas. Asimismo realizaron un trabajo concreto de investigación para dar respuesta a sus preguntas. En este grupo emergieron y se trabajaron conocimientos relacionados con la metodología: talleres, proyectos, trabajo cooperativo, rincones, etc. y en definitiva el método como estrategia (Morin, et al. 2003).

El curso 2010-2011 el proyecto se inició en el nuevo grado de primaria. En el centro de Arte la Panera se mostraba los trabajos que los escolares y otros públicos habían hecho durante el curso anterior a partir de las exposiciones. Volveríamos a tener "Puertas Abiertas" de otras propuestas, de otras obras pero eran los trabajos escolares. Cambiamos de contexto de aprendizaje inicial y visitamos la Fundación Sorigué, que desde 1999 trabaja en la creación de una colección de arte contemporáneo internacional formado por obras que hacen referencia a las diferentes disciplinas. Actualmente está desarrollando el proyecto arquitectónico de un nuevo espacio para la colección. Tiene obras de artistas consagrados como Bill Viola, William Kentridge, Tony Cragg y Jaume Plensa, entre otros.

Apostamos por un trabajo donde tenemos claras las competencias y contenidos a desarrollar relacionados con la materia, pero incorporando los préstamos de conciencia y andamiajes de los contextos de aprendizaje artísticos.

\section{El encaje contemporáneo}

"La punta contemporánea se caracteriza por la utilización del color, de diferentes materiales además del hilo, como metales, papel, plástico. Los diseños llegan a obtener una gran plasticidad, llegando a la tridimensionalidad en diferentes ocasiones. Trabajar la punta contemporánea es pintar, esculpir con hilos. Verdaderas obras de arte (Fig. 4)."

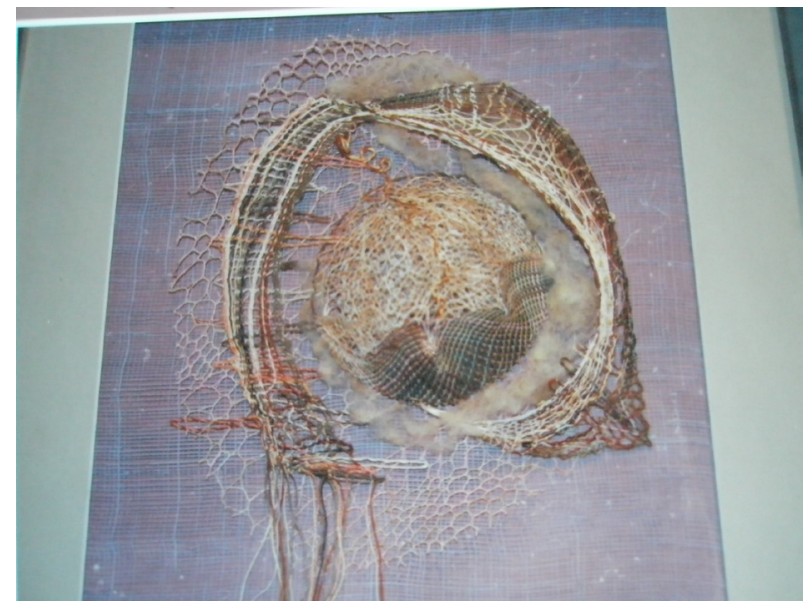

Figura 4. Incògnita (ecografia d'un fetus), Caterina Castellà, 2009. Foto cedida por la autora. 
¿Cuál es la situación actual y la continuidad del proyecto? Del entretejido han emergido valoraciones de las distintas acciones y procesos realizados con el fin de analizar, investigar y crear conocimiento en base a nuestro proyecto.

- Los estudiantes de magisterio, psicopedagogía y de doctorado realizan un seguimiento del espacio híbrido que se realizan en la escuela Príncep de Viana. La recolección de información se lleva a cabo mediante diarios de notas durante la observación participativa. Posteriormente, se sistematiza a partir de un proceso de categorización y triangulación con la información recogida por los diferentes profesionales que intervienen en el proceso. Durante estos años se ha evidenciado reiteradamente la importancia y las posibilidades que permite el trabajo en red para el cambio cualitativo como podemos observar en el siguiente comentario de la Maestra 2: "esta actividad determinada del Proyecto Àlber es posible llevarla a cabo por el trabajo en red: las aportaciones de la Panera, los maestros, técnicos de educación, estudiantes, etc. Porque uno no puede verlo todo. Es un liderazgo compartido que va variando en función de la actividad. Es importante dejar un espacio para que emerja la heterogeneidad desde nuestras competencias".

- Esta experiencia ha coincidido con un estudio que realiza la Fundación Jaume Bofill de Barcelona. Evaluaron nuestro proyecto a partir de entrevistas y grupos de discusión realizados con los profesionales y estudiantes que han participado en el espacio híbrido. Esta evaluación se realiza en el marco del "Proyecto Trama", proyecto interuniversitario impulsado y financiado por la Fundación Jaume Bofill (2009), con la intención de fomentar la construcción de conocimiento sobre la construcción de competencias para el trabajo en red. La pregunta clave que nos planteamos es ¿cómo podemos facilitar en la formación inicial de los profesionales de la educación el desarrollo de estas competencias? Se extrajeron unas conclusiones como guía para la concreción de proyectos de investigación en relación al trabajo en red promovido desde la Universidad.

- La metodología se concretó durante todo el curso. Los estudiantes lo "viven" sin ninguna explicación previa del proyecto. Iniciamos la docencia a partir de la visita al Centro de Arte y en el marco del Proyecto Educ-arte. Y no fue hasta el final de curso que los estudiantes leyeron el artículo (Jové, et al., 2009) donde se explicaba el proyecto. ¿Qué nos dicen sus trabajos escritos?: La estudiante 2 dice: "me he dado cuenta que todo este proceso lo hemos vívido como una experiencia y por eso lo he entendido y lo he conectado todo". La estudiante 5 comenta: "al leer el artículo Educ-arte me siento muy identificada ya que habla de las relaciones que se establecen entre los recursos comunitarios, los centros educativos y la Universidad de Lleida. Creo que en el momento actual formo parte de todos". El estudiante 8 dice: "mitrabajo lo veo comouna obra coral que hemos creado entre todos, durantetodoel curso. Lospréstamos de conciencia han tomadoforma apartir delaorquestaciónpolifónicadetodosloselementosquehanparticipado:profesores, compañeros/as, artistas, contextos de aprendizaje, historia de vida, prácticas..." La estudiante 24 explica: "sin el trabajo en red entre la Panera, la Universidad 
y la escuela mi mirada quizás no se hubiera reeducado, estoy abriendo miradas hacia una enseñanza diferente, funcional... y puedo entender propuestas como las que presentó la estudiante 22 , donde la educación artística es herramienta de expresión y evaluación de contenidos y aprendizajes que se han realizado en otras asignaturas.

Si nos fijamos en los textos utilizan la primera persona del singular y del plural. Todas las voces de los estudiantes muestran un sentimiento de pertenencia al proyecto Educ-arte y se sienten reconocidos en el texto: "somos nosotros" "nosotros lo hemos hecho". Ello nos evidencia que nos movemos en el plano de la coherencia y la consistencia entre lo que decimos y las condiciones de enseñanza y aprendizaje que promovemos para que sean vividas de forma experiencial.

El texto lo leímos todos los participantes y en una sesión del espacio híbrido comentaron:

Maestro 6: "llevo tres años en el proyecto y leer este texto me hace tomar conciencia de lo que estamos haciendo."

Maestra 10 dice: "yo llevo ocho años y leerlo me ha permitido reencauzar mi docencia."

Quizás sea esta una metodología de trabajo para mejorar nuestra docencia: crear las condiciones de aprendizaje para vivirlas, para experienciarlas, escribir sobre ello y favorecer con el texto encuentros polifónicos. Nuestras voces, nuestras vivencias, nuestros sentimientos de identificación y pertinencia en el proyecto legitiman y validan la metodología utilizada desde el Ágora pública y muestran coherencia y consistencia. Según Deleuze \&Guattari (1987) la consistencia lleva al rizoma, ello nos posibilita entretejer la red desde planteamientos rizomáticos.

- El proyecto que hemos presentado está estructurado en base a algunas aportaciones (Civís, Longàs y Riera, 2007) desde la corresponsabilidad real de todos los agentes implicados. El objetivo es entretejer rizomáticamente las relaciones entre la Universidad, la escuela y los recursos comunitarios, especialmente culturales. En todo momento se ha ido desarrollando desde el liderazgo compartido entre los participantes, de manera que en un momento determinado puede adquirir un mayor protagonismo un profesional del centro de arte, o un maestro del centro, o un estudiante... dependiendo en parte de lo que se vaya concretando en el proceso. Hablamos... Profesional del Centro de Arte: "Los estudiantes que han participado, cuando están en el mundo laboral utilizan los recursos del centro de arte con sus alumnos. Los niños de la escuela vienen los fines de semana con sus familias y los estudiantes de la Facultad vienen con amigos". Los maestros de la escuela: "Vemos los recursos comunitarios y sabemos construir y desarrollar el currículo en función de éstos". Los estudiantes de la UdL: "Estar en contextos comunitarios nos da una visión diferente y nos ayuda en nuestra formación profesional y personal". La profesora de la UdL: "Estamos entretejiendo el trabajo en red como encaje contemporáneo que se caracteriza por la utilización del color, de diferentes 
materiales además del hilo, como metales, papel, plástico. Los diseños llegan a obtener una gran plasticidad, llegando a la tridimensionalidad en diferentes ocasiones. Trabajar el encaje contemporánea es pintar, esculpir con hilos, verdaderas obras de arte".

Estamos entretejiendo la red en la comunidad desde el principio dialógico de la complejidad y desde la heterogeneidad. Todo forma parte del todo, como un holograma.

La comunicación en el proyecto es abierta y fluida. Además, esta mejora ha sido sostenible, perdura en el tiempo y durante estos 10 años los procesos de innovación y el cambio cualitativo han devenido desde la investigación-acción. El proyecto Educarte se estructura con la pasión, el propósito y la política de cambio (Hargreaves, 2003).

A modo de síntesis, podemos afirmar que el proyecto lo hemos forjado desde la complejidad y planteamientos rizomáticos. El método que concretamos es la estrategia que permite la formación para todos los implicados en el proceso: desde la formación inicial de los estudiantes de la Facultad a la formación continua de los profesores de la Facultad y la escuela y los profesionales del servicio educativo del centro de arte.

En las últimas décadas y desde el ámbito psicopedagógico muchos trabajos ponen énfasis en la necesidad de articular la red desde el enfoque biopsicosocial y educativo, Promulgan la necesidad de trabajar conjuntamente los profesionales de los ámbitos sociales, sanitarios, educativos que de una manera $u$ otra tienen como objetivo mejorar la calidad de vida de las personas.

Nuestra aportación está enmarcada en los planteamientos socioconstructivistas que sitúan la cultura como elemento básico para la humanización y la calidad de vida de las personas. Es por ello que en nuestro entretejer situamos los recursos comunitarios culturales en general y museos y centros de arte en particular como parte esencial del trabajo en red.

\section{Referencias}

Barab, S. \& Duffy, T. (2000). From practice fields to communities of practice. En Jonassen, D. \& Land, S. (Coords.), Land Theoretical foundations of learning environments (25-26). New York: Routledge.

Civis, M., Longàs, E., Longàs, J. \& Riera, J. (2007). Educació, territori i desenvolupament comunitari. Pràctiques emergents. Revista d'Educació Social, $36,13-23$.

Deleuze, G. \& Guattari, F. (1987). Mil mesetas, capitalismo y esquizofrenia. Valencia: Pre-Textos.

Dewey, J. (2008). El arte como experiencia. Madrid: Paidós.

Eisner, E. (2004). El arte y la creación de la mente: el papel de las artes visuales en la transformación de la conciencia. Barcelona: Paidós.

Fundació Jaume Bofill (2009). Declaració per garantir la formació inicial dels professionals de l'educació per al treball en xarxa. Recuperado de http://www. fbofill.cat/intra/fbofill/documents/declaracio_trama.pdf 
Gadner, H. (2001). El Proyecto Spectrum. Tomo I: Construir sobre las capacitades infantiles. Madrid: Morata.

Hargreaves, A. (comp.) (2003). Replantear el cambio educativo. Un enfoque renovador. Buenos Aires - Madrid: Amorrortu.

Huerta, Ricardo: Maestros, museos y artes visuales. Construyendo un imaginario educativo. Arte, individuo y sociedad, 23, 1, 2011. doi:10.5209/rev_ARIS.2011. v23.n1.5; http://dx.doi.org/10.5209/rev_ARIS.2011.v23.n1.5

Jové, G., Ayuso, H, Sanjuan, R. Vicens, L, Cano, S. \& Zapater, A. (2009). EDUC... arte. Un proyecto de trabajo en red entre universidad, centro de arte y centros educativos. En Huerta, R. y Romà, R. (Eds.), Mentes sensibles. Investigar en educación y en museos (127-138). Valencia: Universidad de Valencia.

Jové, G. \& Betrián, E. (En prensa). Educ- Arte en la Complejidad. Un trabajo en red: la universidad, la escuela y el museo. En Rodríguez, L. (Ed.), La emergencia de los enfoques de la complejidad en América Latina: desafios, contribuciones y compromisos para abordar los problemas complejos del siglo XXI. Buenos Aires: Comunidad de Pensamiento Complejo.

Jové, G., Betrián, E., Ayuso, H. \& Sansó, C. (2010). Proces-Arte y Trans-Formación de maestros. En Pérez, A. I. (Coord.), Reinventar la profesión docente. Libro de Actas. Mesa 9 (80-95). Málaga: AUFOP.

Jové, G., Vicens, L., Cano, S., Serra, O. \& Rodríguez, J. (2006). Desig d'alteritat. Programa Àlber: una eina per atendre a la diversitat a l'aula. Lleida: Pagès editors.

Lenz-Taguchi, H. (2010). Going beyond the theory/practice divide in Early Childhood. Introducing and intra-activite pedagogy. London: Routledge

Morin, E. , Roger, E. \& Domingo, R. (2003). Educar en la Era Planetaria. Barcelona: Gedisa.

Moss, P. (2006). Bringing Politics into the Nursery: Early Childhood Education as a Locus for Democratic Practice. En 16th Conference EECERA. Recuperado de http://www.congress.is/eecera2006/default.asp?q1=keynote.htm

O'Sullivan, S. (2006). Art encounters Deleuze and Guattari Thought Beyond Representation. London: Palgrave Macmillan.

Subirats (1999). Escuela y territorio. El concepto de escuela comunidad. Aula de Innovación Educativa, 79, 43-46.

Zeichner, K. (2010). New epistemologies in teacher education. Rethinking the connections between campus courses and field experiences in college and university-based teacher education. Revista Interuniversitaria de Formación del Profesorado, 68, 123-149. 